TRABAJOS ORIGINALES

\title{
Dieta del Suri, Rhea pennata (Orbigny, 1834) (Aves: Rheidae), en ecosistemas altoandinos de Moquegua, Perú
}

\section{The Lesser Rhea diet, Rhea pennata (Orbigny, 1834) (Aves: Rheidae), in high Andean ecosystems from Moquegua, Peru}

\section{Marli Echaccaya ${ }^{1}$, César Arana ${ }^{1,2 *}$ y Letty Salinas ${ }^{1,3}$}

1 Universidad Nacional Mayor de San Marcos, Facultad de Ciencias Biológicas.

2 Universidad Nacional Mayor de San Marcos, Departamento de Ecología del Museo de Historia Natural

3 Universidad Nacional Mayor de San Marcos, Departamento de Ornitología del Museo de Historia Natural

* Autor para correspondencia

E-mail Marli Echaccaya: maechaccaya@gmail.com

E mail César Arana (autor de correspondencia): caranab@unmsm.edu.pe

E-mail Letty Salinas: Isalinass@unmsm.edu.pe

\begin{abstract}
Resumen
Se estudió la dieta del suri Rhea pennata en la región de Moquegua al sur de Perú, mediante el análisis de heces. Esta especie considerada en "Peligro Crítico" en el Perú se mostró como estrictamente herbívora, principalmente folívora. Se identificaron 44 morfoespecies de plantas en su dieta, de las cuales Oxychloe andina (una especie de Juncaceae dominante en bofedales disturbados) presentó los mayores valores relativos de densidad, peso, volumen y frecuencia. A pesar de la aparente selectividad, esta especie presenta una dieta muy diversa que le permitiría adaptarse a las carencias nutricionales en el hábitat que ocupa. Los datos obtenidos en diferentes hábitats que ocupa el Suri, muestran que en zonas más áridas existe la tendencia a una mayor selectividad debido a la presencia de plantas con compuestos secundarios (fenoles, terpenos y alcaloides) para evitar la herbivoría, mientras que en ambientes con bofedales (como el del presente estudio) la selectividad disminuye, tendiendo a alimentarse de las especies dominantes.
\end{abstract}

Palabras clave: Herbivoría; especies amenazadas; análisis de fecas; Puna; Oxychloe andina; forrajeo selectivo.

\section{Abstract}

We study the Lesser Rhea (Rhea pennata) diet in the Moquegua region of southern Peru, using analysis of feces. This species considered "Critically Endangered" in Peru, was shown as strictly herbivorous, mainly folivorous. A total of 44 plant morphospecies were identified in the diet, of which Oxychloe andina (a Juncaceae species dominant in disturbed wetlands of the Puna) had the highest values of relative density, weight, volume and frequency. Despite the apparent selectivity, this species presents a very diverse diet that would allow it to adapt to the nutritional deficiencies in the habitat that it occupies. The data obtained in different habitats of the Lesser Rhea show that in more arid zones there is a tendency for greater selectivity due to the presence of plant with secondary compounds (phenols, terpenes and alkaloids) to avoid herbivory, while in environments with wetlands (as the present study) the selectivity decreases, tending to feed on the dominant species.

Keywords: Herbivory; threatened species; feces analysis; Puna; Oxychloe andina; selective foraging; Puna Rhea.

Citación:

Echaccaya M., C. Arana y L.Salinas. 2017. Dieta del "Suri”, Rhea pennata (Orbigny, 1834) (Aves: Rheidae), en ecosistemas altoandinos de Moquegua, Perú. Revista peruana de biología 24(2): 139 - 144 (Julio 2017). doi: http://dx.doi.org/10.15381/rpb.v24i2.13491

$\begin{array}{ll}\text { Presentado: } & 25 / 02 / 2016 \\ \text { Aceptado: } & 21 / 04 / 2017\end{array}$

Aceptado: 21/04/2017

Publicado online: $20 / 07 / 2017$
Información sobre los autores:

ME realizó los análisis e identificación de la mayoría de las muestras y preparo el primer borrador del manuscrito, CA y LS realizaron el diseño del muestreo y las evaluaciones de campo, participaron en el análisis de la información y en la elaboración del manuscrito.

Los autores no incurren en conflictos de intereses.

Fuentes de financiamiento: Vicerectorado de Investigación y Posgrado de la Universidad Nacional Mayor de San Marcos; Ministerio de Agricultura y Riego. 


\section{Introducción}

El suri, Rhea pennata (Orbigny, 1834), es una especie de ave de distribución restringida a Chile, Argentina, Bolivia y Perú, considerada clave en los ecosistemas altoandinos que ocupa. Su presencia define áreas importantes para la conservación de aves y biodiversidad en general (Franke et al. 2005). En el Perú, la distribución del Suri se restringe a las zonas altoandinas de las Regiones de Moquegua, Tacna y sur de Puno (Villanueva 2005), habitando planicies de puna desértica y tolares sobre los $3800 \mathrm{~m}$ de altitud (Plenge 1982). Esta especie es considerada en "peligro crítico" en el Perú, según el Decreto Supremo 04-2014-MINAGRI. Sin embargo, existe escasa información en el territorio peruano sobre el estado poblacional, biología y reproducción de la especie (Lleellish et al. 2007, SERFOR 2015).

El conocimiento de los componentes de la dieta y de las estrategias de alimentación en animales silvestres es fundamental para los programas de conservación (Robbins 1993, BirdLife International 2016). Es por esto que se han estudiado los hábitos alimenticios y hábitat de Rhea pennata, principalmente en Argentina, donde su dieta está compuesta por hierbas, arbustos, cactáceas y pastos (Cajal 1988, Bonino et al. 1986, Paoletti \& Puig 2007, Puig et al. 2013, Marinero et al. 2017). En Chile, el hábitat que más utiliza es el bofedal, seguido de los tolares, y su dieta está compuesta principalmente por Oxychloe andina (Galáz 1998, Diaz \& Cardozo 2007). Estudios en el Perú, para las regiones de Puno y Tacna, resaltan la naturaleza herbívora del suri (Montes de Oca y Moreno 2002, Ramos y Galvan 2001), además de la presencia de especies como Oxychloe andina, Distichia muscoides y Austrocylindropuntia floccosa en tolares (Lleellish et al. 2007); y los géneros Hypochaeris, Eleocharis y Calamagrostis (Ramos \& Galvan 2001).

En el presente estudio, se analizaron fecas de Rhea pennata colectadas en el año 2008 en el departamento de Moquegua con el objetivo de conocer los componentes alimenticios y determinar las plantas de importancia en su dieta y contribuir a establecer las bases para el manejo de hábitats que permita la conservación de esta especie en el Perú.

\section{Material y métodos}

Área de estudio.- El área de estudio comprendió nueve localidades, ubicadas en los distritos de Carumas y Chojata en la zona altoandina de la Región de Moquegua (Fig. 1), situada en los Andes occidentales del sur, por encima de los $4000 \mathrm{~m}$, correspondiendo al ecosistema altoandino de Puna (Young et al. 1997). El área está dominada por pajonales de puna (formaciones de gramíneas de hojas duras, rígidas y punzantes dispuestas en manojos aislados distribuidos uniformemente y que en general se les denomina "ichu") y tolares (matorral relativamente ralo, con arbustos que alcanzan $1.5 \mathrm{~m}$ de alto, constituidos principalmente por Asteráceas y numerosas hierbas anuales, arbustos como Parastrephia quadrangularis conocido como "tola" y Parastrephia lucida de hojas aciculares que predomina a niveles superiores hasta $4900 \mathrm{~m}$ (Weberbauer 1945, Montesinos 2011).

\section{Colecta y análisis de muestras}

La colecta de fecas fue realizada en junio de 2008 en el marco del Primer Censo Nacional del Suri con un total de cuatro estaciones de muestreo, en las cuales se colectaron 14 fecas. Las muestras fueron preservadas en sobres con papel secante, para ser derivadas a la Universidad Nacional Mayor de San Marcos; en el laboratorio se preservaron en alcohol al 70\% y durante los años 2010 a 2012 se examinaron siguiendo los pasos sugeridos por Rosenberg y Cooper (1990). Para determinar la composición de la dieta, se analizó la morfología de las partes de las plantas y se realizó un análisis microhistológico de los componentes encontrados.

Identificación de las especies vegetales en la dieta del suri.Los componentes clasificados en raíces, ramas, hojas, flores, frutos, semillas, fibras y otras estructuras no vegetales como piedras y sedimento se agruparon por similitud, guardándolas en sobres de papel glassine y codificándolos para su identificación a nivel específico.

Se preparó una colección de referencia con el material vegetal mejor conservado encontrado en las fecas. Este material se identificó hasta el mayor nivel taxonómico posible empleando bibliografía especializada y comparando la morfología de los fragmentos con la ayuda de un microscopio estereoscopio. Para verificar la morfología de algunas hojas, ramas y frutos y conocer la forma de vida de las especies de plantas, se consultó las colecciones del Herbario San Marcos (USM) del Museo de Historia Natural de la Universidad Nacional Mayor de San Marcos.

Cuando las muestras no pudieron identificarse mediante la comparación morfológica macroscópica, se usaron técnicas de análisis microhistológico. Para ello, se prepararon muestras de referencia de especies vegetales colectadas en zonas cercanas al área estudio y muestras de las hojas presentes en las fecas. El procedimiento siguió los estándares de las técnicas en anatomía foliar (Cutler 1987, D'Ambrogio 1986): las hojas se sumergieron en agua caliente con detergente por 5 minutos y/o se hirvieron, se hicieron cortes transversales y longitudinales en la parte media de las hojas, se colocaron en láminas portaobjetos en un medio de safranina y glicerina para visualizarlas al microscopio. Se usó

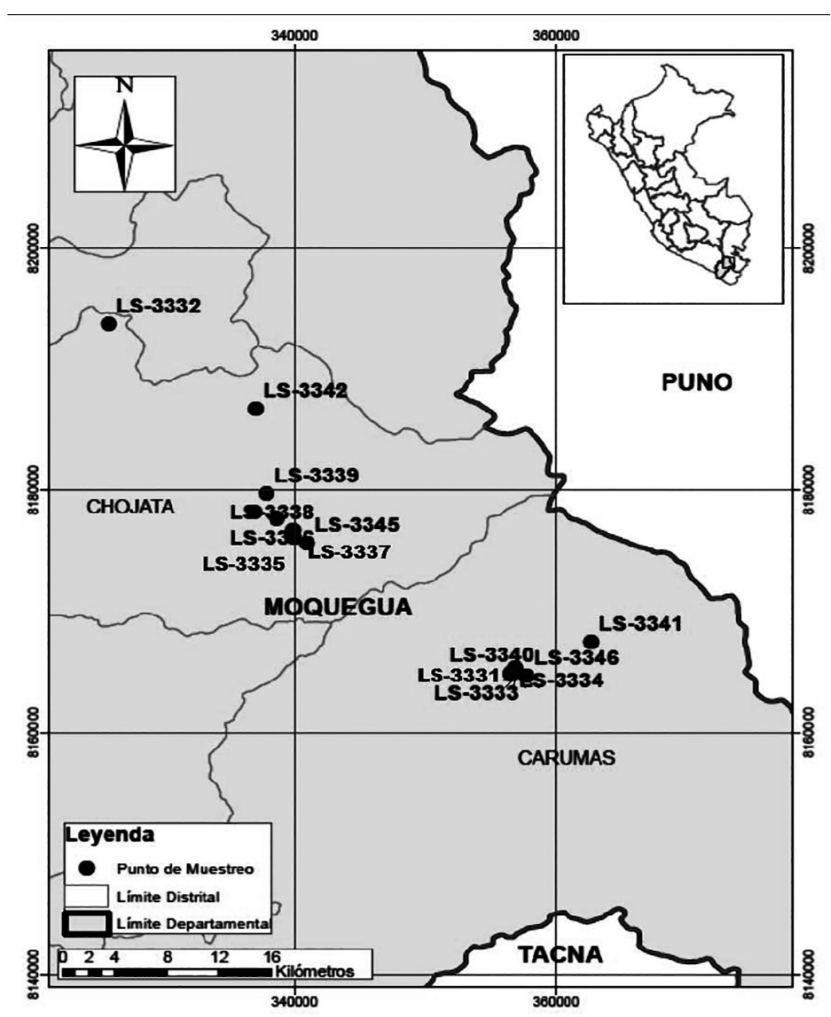

Figura 1. Ubicación de los puntos de muestreo de las fecas del suri Rhea pennata analizadas, Región de Moquegua, Perú. 
bibliografía especializada para la identificación de los cortes anatómicos transversales y longitudinales de las hojas (Brown 1958, Metcalfe 1960, Metcalfe 1969, Stancík 2003, GómezSánchez y Tellez-Pimienta 2008).

Determinación de los hábitos alimenticios.- Se determinó el número de fragmentos, peso $(\mathrm{g})$ y volumen $\left(\mathrm{mm}^{3}\right)$ para cada componente (especie o morfoespecie), los datos obtenidos se halló la densidad relativa (número de fragmento de cada especie sobre el número total de fragmento), el peso relativo (peso de cada ítem sobre el peso total), el volumen relativo (volumen de cada ítem sobre el volumen total de la muestra), y la frecuencia (número de muestras con el ítem sobre el número total de muestras), y se calculó el Índice de Valor de Importancia (IVI) de los componentes alimentarios (Rosenberg \& Cooper 1990, Cornejo \& Jiménez 2001, Salinas 2001):

\section{$I V I=$ Densidad relativa + Peso relativo + Volumen relativo + Frecuencia}

Finalmente, para conocer el grado de variabilidad del alimento utilizado por el ave, se calculó la amplitud de su nicho trófico usando el Î́ndice de Levins (B) y su valor estandarizado (B), según el cual un individuo será "generalista" si la especie consume los diferentes recursos alimenticios en la misma proporción, alcanzando valores de hasta uno, al contrario, un individuo será "especialista" si los individuos se alimentan preferentemente de un único tipo de alimento, siendo sus valores cercanos a cero (Krebs 1989).

\section{Resultados y discusión}

Se encontraron 50 componentes en el análisis de las fecas. Estos componentes son orgánicos e inorgánicos. Los componentes orgánicos (49) representaron el $96,11 \%$, y son en su mayoría componentes vegetales $(96 \%)$ y en menor porcentaje animales, en especial Lepidoptera $(0.11 \%)$. Estos resultados indicarían una dieta herbívora y son similares a los obtenidos en estudios anteriores sobre la dieta de Rhea pennata a través de fecas (Bonino et al. 1986; Ramos \& Galvan 2001, Montes de Oca \& Moreno 2002, Lleellish et al. 2007; Cajal 1998, Paoletti $\&$ Puig 2007, Marinero et al. 2017). Los escasos artrópodos hallados en la dieta de esta especie (Noble 1991, Lleellish et al. 2007, Paoletti \& Puig 2007), moscas o en nuestro estudio Lepidópteros, podrían ser considerados como accidentales, encontrados en las fecas por arribo posterior a la deposición, al estar expuestas al ambiente. Esta misma ausencia de insectos ha sido reportada en Argentina y atribuida a la escasez de éstos en la zona altoandina (Marinero et al. 2017).

Los componentes inorgánicos fueron piedras con un 3.89\% del volumen total, las que variaron de $2 \mathrm{~mm}$ de diámetro hasta 30 x $15 \mathrm{~mm}$, encontrándose en una frecuencia menor a la reportada por Lleellish y colaboradores (2007) quienes reportan hasta un $25.6 \%$ de ellas. Se tiene poca información del rol que tienen las piedras en el género Rhea, pero a nivel de Struthioniformes en general se sabe que pueden usarlas como ayuda mecánica para procesar la materia vegetal gruesa de la cual se alimentan, pudiendo transportar hasta $907 \mathrm{~g}$ de piedrecillas en su estómago de unos $2.5 \mathrm{~cm}$ de diámetro (Noble 1991).

Los componentes vegetales fueron identificados hasta morfoespecie ( 44 tipos) y se agruparon en 17 familias, entre las que destacaron Poaceae, (13 especies), Cyperaceae (5) y Asteraceae (4) (Tabla 1). Los géneros más representativos de la familia Poaceae fueron Poa, Festuca y Calamagrostis. La familia Cyperaceae estuvo representada por el género Carex y algunos especímenes no identificados. La familia Asteraceae estuvo representada por los géneros Senecio e Hypochaeris.

Las especies herbáceas representaron un $84 \%$ del volumen total, el porcentaje restante corresponde a arbustos, entre los que podemos mencionar a Senecio spinosus, Tetraglochin sp. y una Cactaceae indeterminada. La composición, a nivel de familias, fue similar a la mencionada por Montes de Oca y Moreno (2002) y correspondió a lo reportado para la flora en estudios próximos al área de evaluación, en la cual predominaron las familias Asteraceae $(15 \%$ a $24 \%)$ y Poaceae $(5.5 \%$ a $11 \%)$ principalmente (Montesinos 2011, Arakaki \& Cano 2003).

Las estructuras vegetales con mayores valores de volumen fueron las hojas (32\% del total), seguido de frutos (24\%) y restos vegetales no identificables (19\%). El resto de estructuras (como ramas, fibras, semillas, tallos, flores y plantas completas) representaron menos del 10\% cada uno. Estos resultados son similares a lo encontrado en otros estudios con el mayor porcentaje de estructuras vegetales representado por hojas y semillas (Paoletti \& Puig 2007, Puig et al. 2013, Marinero et al. 2017).

De las 44 morfoespecies de plantas identificadas, Oxychloe andina (64.2\%), Festuca spp. (6.9\%), Cyperaceae Indet.1 (4.4\%) y Poa spp. (3.3\%), tuvieron los mayores valores de densidad relativa. Oxychloe andina (63.0\%), Festuca sp.1 (6.4\%), Poa spp. (4.8\%) y Sisyrinchium sp. (3.8\%) tuvieron los mayores pesos relativos; mientras que Oxychloe andina (41.7\%), Festuca spp. (13.4\%), Poa spp. (12.9\%), Cyperaceae Indet. (8.5\%) y Distichia muscoides (5.8\%), presentaron los mayores valores de volumen relativo. Finalmente, una vez más Oxychloe andina presentó la mayor frecuencia relativa (100\%), seguida de Poa spp. y Onagraceae Indet. (cada una 86\%), Calamagrostis heterophylla y Silene sp. (cada una 79\%) (Tabla 1, Fig. 2). Este alto número de especies es similar a lo registrado en Argentina (Marinero et al. 2017) y muestra un comportamiento correspondiente a una estrategia de forrajeo generalista (Recher 1990), lo cual es esperado en ambientes con escasos recursos donde las especies presentan dietas flexibles, consumiendo diversos tipos de especies de plantas para cubrir sus requerimientos nutricionales (Puig et al. 1996).

Oxychloe andina ha sido encontrada en otros estudios de dieta de Suri, pero en menor porcentaje, esto podría deberse a que si bien Distichia muscoides es la especie predominante en los bofedales (Weberbauer 1945), Oxychloe andina se ha encontrado predominando en algunos bofedales erosionados (ALT-PNUD 2001). Los estudios de dieta de esta especie en Puno, Tacna y Moquegua (la presente investigacion) coinciden en especies como Oxychloe andina, Hypochaeris taraxacoides, Distichia muscoides, Poa spp, Agrostis sp., Calamagrostis spp. y Ciperáceas. Sin embargo, en este estudio fue evidente la ausencia de Austrocylindropuntia floccosa registrada por los otros estudios, donde se indica que consume las estructuras de tallo y frutos principalmente, esta ausencia podría deberse a que en la época de colecta de fecas (junio) no presenta flores ni frutos (según lo indica Herrera (1924) y los ejemplares de herbarios consultados: USM, Neotropical Herbarium Specimens). Otra especie ausente, aunque es comúnmente mencionada por la población local como parte de la dieta del Suri, es la tola (Villanueva 2005). Como tola se denominan a especies de hábito arbustivo del género Parastrephia (como Parastrephia lucida y Parastrephia quadrangularis) que se encuentran en las formaciones vegetales llamados tolares que llegan hasta los $4500 \mathrm{~m}$ de altitud. No se 
Tabla 1. Componentes vegetales en el análisis de fecas de Rhea pennata. Se muestra los valores relativos de volumen, densidad, peso y frecuencia e índice de valor de importancia (IVI). Oxychloe andina (Juncaceae), Poa spp. y Festuca spp. (Poaceae), Onagraceae (indeterminada) y Silene sp. (Caryophyllaceae) son las especies con mayores valores de IVI.

\begin{tabular}{|c|c|c|c|c|c|c|c|}
\hline $\begin{array}{l}\text { Familia } \\
\text { Especie (morfoespecie) }\end{array}$ & $\begin{array}{l}\text { Forma de } \\
\text { vida }\end{array}$ & Estructura & $\begin{array}{l}\text { Volumen } \\
\text { relativo }\end{array}$ & $\begin{array}{l}\text { Densidad } \\
\text { relativa }\end{array}$ & $\begin{array}{l}\text { Peso } \\
\text { relativo }\end{array}$ & $\begin{array}{l}\text { Frecuencia } \\
\text { relativa }\end{array}$ & IVI \\
\hline \multicolumn{8}{|l|}{ Amaranthaceae } \\
\hline Amaranthaceae Indeterminado & Hierba & Semilla & 0.02 & 0.08 & 0.53 & 28.57 & 29.21 \\
\hline \multicolumn{8}{|l|}{ Apiaceae } \\
\hline Oreomyrrhis andicola & Hierba & Fruto (Mericarpo) & 0.07 & 0.57 & 0.46 & 35.71 & 36.82 \\
\hline Lilaeopsis macloviana & Hierba & Hoja & 0.43 & 0.29 & 0.42 & 21.43 & 22.57 \\
\hline \multicolumn{8}{|l|}{ Asteraceae } \\
\hline Hypochaeris taraxacoides & Hierba & Hoja. Flor & 1.82 & 1.33 & 0.32 & 42.86 & 46.33 \\
\hline Senecio spinosus & Arbusto & Rama (espina) & 0.07 & 0.15 & 0.04 & 35.71 & 35.98 \\
\hline Asteraceae Indeterminado 1 & & Fruto & 0.04 & 0.03 & 0.12 & 21.43 & 21.63 \\
\hline Asteraceae Indeterminado 2 & & Fruto & 0.02 & 0.14 & 0.10 & 14.29 & 14.55 \\
\hline \multicolumn{8}{|l|}{ Brassicaceae } \\
\hline Lepidium sp. & Hierba & Semilla. Fruto & 0.14 & 0.16 & 0.82 & 57.14 & 58.27 \\
\hline \multicolumn{8}{|l|}{ Bryophyta } \\
\hline Bryophyta Indeterminado & Hierba & Planta completa & 0.39 & 0.24 & 0.31 & 71.43 & 72.36 \\
\hline \multicolumn{8}{|l|}{ Cactaceae } \\
\hline Cactaceae Indeterminado & Arbusto & Semilla & 0.07 & 0.08 & 0.27 & 42.86 & 43.27 \\
\hline \multicolumn{8}{|l|}{ Campanulaceae } \\
\hline Hypsela reniformis & Hierba & Flor & 0.00 & 0.00 & 0.00 & 14.29 & 14.29 \\
\hline \multicolumn{8}{|l|}{ Caryophyllaceae } \\
\hline Silene sp. & Hierba & Fruto & 0.44 & 1.15 & 1.45 & 78.57 & 81.62 \\
\hline Caryophyllaceae Indeterminado 1 & Hierba & Hoja & 0.01 & 0.01 & 0.06 & 35.71 & 35.80 \\
\hline Caryophyllaceae Indeterminado 2 & Hierba & Semilla & 0.03 & 0.03 & 0.60 & 50.00 & 50.67 \\
\hline \multicolumn{8}{|l|}{ Cyperaceae } \\
\hline Carex sp. & Hierba & Hoja & 4.20 & 2.61 & 2.53 & 21.43 & 30.77 \\
\hline Cyperaceae Indeterminado 1 & Hierba & Hoja & 8.54 & 4.42 & 1.82 & 21.43 & 36.21 \\
\hline Cyperaceae Indeterminado 2 & Hierba & Hoja & 0.91 & 1.24 & 1.00 & 7.14 & 10.30 \\
\hline Cyperaceae Indeterminado 3 & Hierba & Hoja & 0.57 & 0.36 & 0.66 & 21.43 & 23.02 \\
\hline Cyperaceae Indeterminado 4 & Hierba & Fruto & 0.03 & 0.05 & 0.18 & 21.43 & 21.69 \\
\hline \multicolumn{8}{|l|}{ Fabaceae } \\
\hline Astragalus sp. & Arbusto & Semilla & 0.00 & 0.00 & 0.01 & 21.43 & 21.44 \\
\hline Familia y especie indeterminada & & Fruto & 0.03 & 0.02 & 0.18 & 21.43 & 21.66 \\
\hline \multicolumn{8}{|l|}{ Iridaceae } \\
\hline Sisyrinchium sp. & Hierba & Fruto. Semilla & 1.02 & 1.30 & 3.81 & 71.43 & 77.56 \\
\hline \multicolumn{8}{|l|}{ Juncaceae } \\
\hline Distichia muscoides & Hierba & Hoja & 5.79 & 1.19 & 0.36 & 35.71 & 43.05 \\
\hline Juncus subgen. Poiophylli & Hierba & Fruto & 0.30 & 0.43 & 0.07 & 35.71 & 36.51 \\
\hline Oxychloe andina & Hierba & Hoja. Fruto. Semilla & 41.76 & 64.19 & 63.01 & 100.00 & 268.95 \\
\hline \multicolumn{8}{|l|}{ Malvaceae } \\
\hline Nototriche sp. & Hierba & Fruto (Mericarpo) & 0.00 & 0.01 & 0.07 & 14.29 & 14.37 \\
\hline Malvaceae Indeterminado & Hierba & Fruto (Mericarpo) & 0.00 & 0.00 & 0.00 & 7.14 & 7.15 \\
\hline \multicolumn{8}{|l|}{ Onagraceae } \\
\hline Onagraceae Indeterminado & Hierba & Semilla & 1.06 & 0.49 & 3.07 & 85.71 & 90.33 \\
\hline Poaceae & & & & & & & \\
\hline Agrostis sp. & Hierba & Hoja & 2.12 & 2.84 & 1.30 & 42.86 & 49.12 \\
\hline Calamagrostis heterophylla & Hierba & Flor & 0.17 & 0.13 & 0.08 & 78.57 & 78.96 \\
\hline Calamagrostis spp. & Hierba & Hoja. Flor & 0.93 & 1.65 & 2.00 & 50.00 & 54.59 \\
\hline Dissanthelium sp. & Hierba & Flor & 0.37 & 1.25 & 0.39 & 64.29 & 66.29 \\
\hline Dissanthelium trollii & Hierba & Flor & 0.34 & 0.17 & 0.08 & 35.71 & 36.30 \\
\hline Festuca spp. & Hierba & Hoja. Flor & 13.37 & 6.89 & 6.41 & 71.43 & 98.10 \\
\hline Muhlenbergia tenuissima & Hierba & Flor & 0.06 & 0.05 & 0.05 & 7.14 & 7.31 \\
\hline Poa gymnantha & Hierba & Hoja & 0.49 & 0.87 & 0.34 & 7.14 & 8.84 \\
\hline Poa spp. & Hierba & Hoja. Flor & 12.88 & 3.34 & 4.80 & 85.71 & 106.73 \\
\hline Festucoide Indeterminado & Hierba & Hoja & 1.30 & 2.11 & 1.94 & 28.57 & 33.92 \\
\hline Pooideae Indeterminado & Hierba & Hoja & 0.15 & 0.08 & 0.21 & 7.14 & 7.59 \\
\hline Poaceae Indeterminado 1 & Hierba & Fruto & 0.00 & 0.00 & 0.01 & 7.14 & 7.15 \\
\hline Poaceae Indeterminado 2 & Hierba & Fruto & 0.00 & 0.00 & 0.00 & 7.14 & 7.15 \\
\hline Polygonaceae & & & & & & & \\
\hline Rumex sp. & Hierba & Fruto & 0.00 & 0.00 & 0.01 & 7.14 & 7.15 \\
\hline Rosaceae & & & & & & & \\
\hline Alchemilla diplophylla & Hierba & Hoja & 0.01 & 0.02 & 0.10 & 21.43 & 21.56 \\
\hline Tetraglochin sp. & Arbusto & Fruto & 0.01 & 0.01 & 0.00 & 7.14 & 7.16 \\
\hline
\end{tabular}


encontraron indicios de hojas, flores o semillas de estas especies en el presente estudio, aunque algunos de los restos de ramas podrían pertenecer a las mencionadas especies de tola.

El índice de diversidad de componentes ( $H^{\prime}$ de ShannonWinner) en la dieta de Rhea pennata, fue de $\mathrm{H}^{\prime}=2.48 \mathrm{bits} / \mathrm{item}$, una diversidad mayor comparando con estudios en Argentina Paoletti y Puig (2007) que encontraron un índice de diversidad en la dieta de 0.77 lo que sugiere un aprovechamiento de la mayor diversidad presente en el área de estudio. La amplitud de nicho (Índice de Levins) fue de 2.45 y el índice estandarizado de Levins de un valor cercano a cero $\left(B_{a}=0.03\right)$ mostrando una reducida amplitud de nicho de alimentación originada por la marcada dominancia de Oxychloe andina, por lo que es recomendable una investigación de la disponibilidad de esta planta en los bofedales de Moquegua, asi como un estudio de su variación estacional.

Este valor de amplitud de nicho además indicaría una dieta herbívora de hábitos alimenticios especialista. Sin embargo, se deben acompañar a estos estudios una relación con la disponibilidad y abundancia de las presas (alimentos) para poder realizar una clasificación de este tipo (Jaksic 1989). Estudios que se realizaron en Argentina tomando en cuenta la disponibilidad de alimentos (Paoletti \& Puig 2007) mencionan para esta especie una amplia variedad de plantas en su dieta por lo que puede considerarse generalista, es decir, una especie que forrajea un elevado número de especies vegetales pertenecientes a diversos tipos de plantas. Sin embargo, los cambios estacionales en el número especies preferidas y evitadas sugieren un aumento del comportamiento selectivo por temporadas (Puig et al. 2013).

Los resultados del presente estudio y lo analizado en las poblaciones de Argentina, muestran diferencias en la composición de especies, que al parecer dependen de las características del hábitat donde se ubican las aves. Por un lado, en zonas cercanas a bofedales (como el presente estudio), el Suri parece aprovechar las especies más dominantes en el mismo, mientras que en zonas más áridas

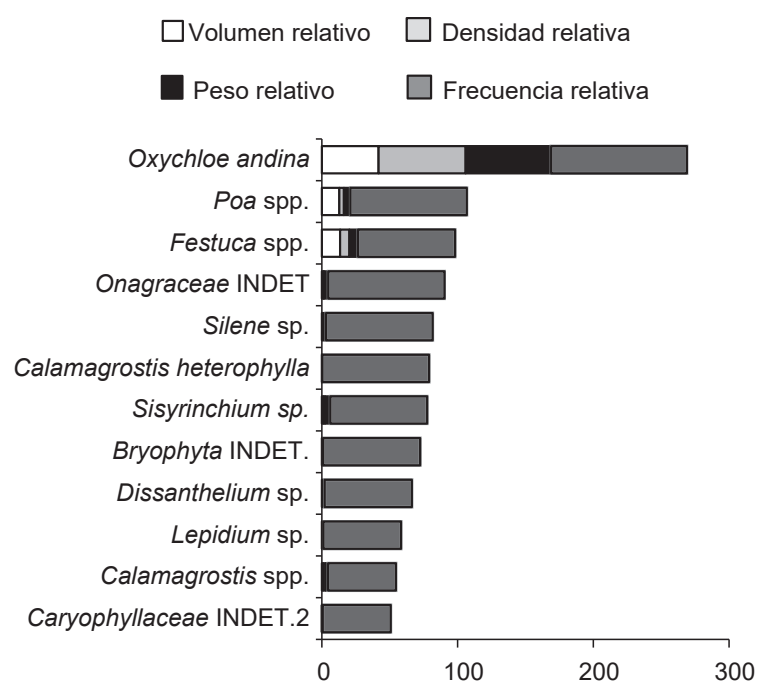

Figura 2. Especies de plantas con mayores índices de valor de importancia (IVI), mostrando los valores relativos de volumen, densidad, peso y frecuencia en la dieta del Suri en Moquegua. Oxychloe andina es por mucho el componente más importante en la dieta del Suri. (como en Argentina) tienen mayor selectividad del alimento, evitando plantas que produzcan grandes montos de metabolitos secundarios (Marinero et al. 2017). En estos ambientes con pocos recursos la selectividad del alimento por parte de los herbívoros se encuentra en un balance entre la disponibilidad de las especies de plantas, el gasto energético que significa el consumir grandes montos de compuestos secundarios y la calidad nutricional de los tejidos vegetales (Freeland \& Janzen 1974, Crawley 1983). Lo que llevaría a pensar que los herbívoros son más selectivos durante los periodos de menor disponibilidad de recursos, debido a una menor proporción de alimentos palatables o con buenas cualidades nutricionales (Weckerly \& Kennedy 1992). En ambientes con poca estacionalidad como los bofedales, la selectividad puede ser escasa para la mayoría de herbívoros.

El Suri al parecer exhibe, sin embargo, cierta selectividad en su comportamiento de forrajeo en áreas sin bofedales (i.e. Marinero et al. 2017), dondemuchas especies abundantes de la puna, presentan muchos metabolitos secundarios (como fenoles, terpenos y alcaloides) para evitar la herbivoría. En las zonas de bofedales, donde plantas más palatables abundan y la vegetación es estable durante todo el año, la selectividad es menor como se observa en nuestro estudio y en otras áreas del sur del Perú.

Rhea pennata es una especie muy importante en el Perú, está incluida en el apéndice I de la CITES (https://cites.org/ esp/app/appendices.php), categorizada como Críticamente amenazada (Decreto Supremo 004-2014-MINAGRI), presenta valor cultural, social y económico además de contar con un Plan Nacional para su Conservación para el periodo 20152020 (SERFOR 2015). Los datos de los censos nacionales de 2008 y 2016, muestran una reducción de la población de 447 a 350 individuos a nivel nacional y de 186 a 72 individuos en Moquegua (Christian Riveros, com.pers.) Los Suris brindan también servicios ambientales, al contribuir potencialmente a la dispersión de semillas de plantas andinas como Sisyrinchium sp. (Echaccaya et al. 2012).

Los resultados del presente estudio contribuirán a la gestión del hábitat del Suri, en especial porque las planicies altoandinas de las regiones donde se distribuye enfrentan fuerte presión antrópica por pastoreo y construcción de infraestructura que ocasionan fragmentación del hábitat. En Moquegua se requiere de un seguimiento de las áreas en las que abundan Oxychloe andina. Si bien en Tacna el Tolar es el hábitat principal para el Suri (Salinas 2007), en Moquegua la conservación de los bofedales, aun los que presentan presión antrópica son de gran importancia como fuente de alimento para esta ave.

Se requiere mayor investigación científica de esta icónica especie amenazada en el Perú. Son necesarias investigaciones sobre disponibilidad, selección y valor nutricional del alimento, así como análisis del paisaje que ocupa para una efectiva gestión de la especie dentro y fuera de áreas de conservación.

\section{Agradecimientos}

Al Servicio Nacional Forestal y de Fauna Silvestre del Ministerio de Agricultura y Riego por el apoyo con el material del censo. Al Vicerectorado de Investigación y Posgrado de la UNMSM por el financiamiento parcial. Al Herbario USM y a los profesores Asunción Cano, Manuel Marín, Mery Suni y Betty Millán por su apoyo en el proceso de identificación de las especies botánicas. 


\section{Literatura citada}

ALT-PNUD (Autoridad Binacional del Lago Titicaca \& el Programa de las Naciones Unidas para el Desarrollo). 2001. Diagnóstico e inventario de recursos naturales de flora y fauna. Proyecto PER/98/G32 Conservación de la Biodiversidad en la Cuenca del Lago Titicaca, Desaguadero, Poopó y Salar de Coipasa (TDPS). Sub Contrato 21.27: "Estudio para establecer nuevos linderos de la Reserva Nacional del Titicaca y elaborar el plan maestro".

Arakaki M. \& A. Cano. 2003. Composición florística de la cuenca del río Ilo-Moquegua y Lomas de Ilo, Moquegua, Perú. Revista Peruana de Biología 10(1): 5-19 http://dx.doi.org/10.15381/ rpb.v10i1.2472

BirdLife International. 2016. Rhea pennata. The IUCN Red List of Threatened Species 2016: e.T22728199A94974489. Downloaded on 27 March 2017.

Bonino N., G. Bonvissuto, A. Pelliza Sbriller, \& R. Solmo. 1986. Hábitos alimentarios de los herbívoros en la zona central del área ecológica Sierras y Mesetas Occidentales de Patagonia. Revista Argentina de Producción Animal 6, 275-287.

Brown W.V. 1958. Leaf anatomy in grass systematics. Bot. Gaz. 119:170178.

Cajal J.L. 1988. The lesser rhea in the Argentina Puna Region: present situation. Biological Conservation 45: 81-91. https://doi. org/10.1016/0006-3207(88)90040-7

CITES (Convention on International Trade in Endangered Species of Wild Fauna and. Flora) 2016.The CITES Appendices. Disponible en <https://cites.org/esp/app/appendices.php $>$

Cornejo A. \& Jiménez P. 2001. Dieta del zorro andino Pseudalopex culpaeus (Canidae) en el matorral desértico del sur del Perú. Revista de Ecología Latino Americana 8: 01-09.

Crawley M.J. 1983. Herbivory. the Dynamics of Animal-Plant Interactions. Blackwell Sci. Publ.: Oxford.

Cutler D.F. 1987. Anatomía Vegetal Aplicada. 1era Edición. Buenos Aires Argentina: Ed. Librería Agropecuaria S.A. 220pp.

D’Ambrogio De Argueso A. 1986. Manual de Técnicas en Histología Vegetal. 1era Edición. Buenos Aires, Argentina: Editorial Hemisferio Sur S.A. 81pp.

Decreto Supremo N ${ }^{\circ}$. 004-2014-MINAGRI. 2014. Decreto Supremo que aprueba la actualización de la lista de clasificación y categorización de las especies amenazadas de fauna silvestre legalmente protegidas. 8 de abril de 2014. El Peruano Normas Legales: 520497- 520504 .

Díaz M.A., \& C. Cardozo. 2007. Plan Nacional de Conservación del Suri Pterocnemia pennata tarapacensis (Chubb, 1913) en Chile. Corporación Nacional Forestal. Stgo. Chile 73 pp.

Echaccaya M., M. Suni, \& C. Arana. 2012. Capacidad germinativa de semillas silvestres preservadas en fecas de "Suri" Rhea pennata Orbigny, 1834. En Libro de Resúmenes del XIV Congreso Nacional de Botánica. Pp. 160. Trujillo 2012.

Franke I., J. Mattos, L. Salinas, C. Mendoza \& S. Zambrano. 2005. Áreas importantes para la conservación de las aves en el Perú. Pp. 471619 in BirdLife International \& Conservation Internacional. Áreas importantes para la conservación de las aves en los Andes Tropicales. Quito, Ecuador: BirdLife Internacional (BirdLife Conservation Series No 14)

Freeland W.J. \& D.H. Janzen. 1974. Strategies in herbivory by mammals: The role of plant secondary compounds. The American Naturalist. 108: 269-289. https://doi.org/10.1086/282907

Galaz J.L. 1998. La conservación del Suri (Pterocnemia pennata tarapacensis, d'Orbigny) en Chile En: Valverde V (ed.) $1998 \mathrm{La}$ Conservación de la Fauna Nativa de Chile. CONAF. 19-25 pp

Gómez-Sánchez M. y Téllez-Pimienta K. 2008. Anatomía foliar de algunas gramíneas alpinas y subalpinas del Eje Volcánico Transversal, México. Journal of The Botanical Research Institute of Texas 2(1): $495-515$

Herrera F.1924. Las Cactáceas de los alrededores dela ciudad del Cuzco. Revista Chilena de Historia Natural 28:31-38.

Jaksic F.M. 1989. Opportunism vs selectivity among carnivorous predators that eat mammalian prey: a statistical test o hypothesis. Oikos 56:427-430 https://doi.org/10.2307/3565630

Krebs C.J. 1989. Ecological Methodology. New York. Harper and Row Publishers. 654 pp.
Lleellish M., L. Salinas y E. Chipana. 2007. Situación del Suri Pterocnemia pennata en el Perú. INRENA. Intendencia Forestal y de Fauna Silvestre. Lima.

Marinero N.V., J.L. Navarro \& M.B. Martella. 2017. Does food abundance determine the diet of the Puna Rhea (Rhea tarapacensis) in the Austral Puna desert in Argentina? Emu - Austral Ornithology: http://dx.doi.org/10.1080/01584197.2016.1277762

Metcalfe C.R. 1960. Anatomy of the Monocotyledons. I. Gramineae. Oxford. 731pp.

Metcalfe C.R. 1969. Anatomy as an Aid to Classifying the Cyperaceae. American Journal of Botany. Vol. 56, No. 7, Special Issue: XI International Botanical Congress Held at the University of Washington (Aug., 1969), pp. 782-790

Montes De Oca V.A. \& E. Moreno. 2002. Bioecología del Suri en la zona altoandina de Tupala-Puno. Revista Universitaria 10: 6-10

Montesinos D. 2011. Diversidad Florística de la cuenca alta del rio TamboIchuña, Moquegua, Perú. Revista Peruana de Biología 18(1): 119-132. https://doi.org/10.15381/rpb.v18i1.156

Noble J.C. 1991. On ratites and their interactions with plants. Revista Chilena de Historia Natural 64: 85-118.

Paoletti G. \& S. Puig. 2007. Diet of the Lesser Rhea (Pterocnemia pennata) and availability of food in the Andean Precordillera (Mendoza, Argentina). Emu 107(1): 52-58.

Plenge M. 1982. The distribution of the lesser Rhea Pterocnemia pennata in southern Peru and northern Chile. The British Ornithologist's Union. Ibis 124: 168-172. https://doi. org/10.1111/j.1474-919X.1982.tb03755.x

Puig S., F. Videla, S. Monge \& V. Roig. 1996. Seasonal variations in guanaco diet (Lama-Guanicoe Muller 1776) and food availability in northern Patagonia, Argentina. Journal of Arid Environments. 34, 215-224. https://doi.org/10.1006/jare.1996.0103

Puig S., M.I. Cona, F. Videla, \& E. Méndez. 2013. Diet selection by the lesser rhea (Rhea pennata pennata) in Payunia, Northern Patagonia (Mendoza, Argentina). Rev. FCA UNCUYO. 2013. 45(1): 211-224.

Ramos L. \& A. Galván. 2001. Composición alimenticia de Pterocnemia pennata "Suri" en dos zonas de Puno en: Libro de resúmenes de la IV Jornada Nacional de Ornitología, Puno. Pág. 19.

Recher H.F. 1990. Specialist or generalist: Avian response to spatial and temporal changes in resources. Studies Avian Biology. 13, 333-336.

Robbins C. 1993. Wildlife feeding and nutrition. New York: Academic Press, Inc. 352p. https://doi.org/10.1016/B978-0-12-5893800.50003-7

Rosenberg K.V. \& R.J. Cooper. 1990. Approaches to avian diet analysis. Studies in Avian Biology 13: 80-90.

Salinas L. 2001. Aves dispersoras de semillas de plantas leñosas del Bosque de Zárate, Lima. Tesis, Biólogo con mención en Zoología. Facultad de Ciencias Biológicas Universidad Nacional Mayor de San Marcos.

Salinas L. 2007. Avifauna de Tacna en la ruta del censo de Suri Pterocnemia pennata. INRENA-Ministerio de Agricultura. Perú.

SERFOR. 2015. Plan Nacional para la Conservación del Suri (Rhea pennata): Periodo 2015-2020. Ministerio de Agricultura y Riego, Lima.

Stancík D. 2003. Las especies del género Festuca (Poaceae) en Colombia. Darwiniana 41(1-4): 93-153

Young K.R., B. León \& A. Cano. 1997. Peruvian Puna. En Davis S., Heyewood V., Herrera-Macbride O., Villa-Lobos J. \& Hamilton A. (Eds.), Centres of Plant Diversity. A Guide and Strategy for their Conservation. Volume 3. The Americas. The World Wide Fund and IUCN-The World Conservation Union. 470-476p.

Villanueva J. 2005. Distribución Actual del Suri Pterocnemia tarapacensis a nivel nacional. Tesis para optar el grado de Magíster Scientiae. Universidad Nacional Agraria la Molina. Lima.

Weberbauer A. 1945. El Mundo Vegetal de los Andes Peruanos. Lima. Estación experimental Agrícola de la Molina, Dirección de Agricultura, Ministerio de Agricultura. 776p.

Weckerly, Floyd W., y Michael L. Kennedy. 1992. Examining hypotheses about feeding strategies of white-tailed deer. Canadian Journal of Zoology 70 (3): 432-39. https://doi.org/10.1139/z92-066 